J. Nonlinear Var. Anal. 4 (2020), No. 1, pp. 87-105

Available online at http://jnva.biemdas.com

https://doi.org/10.23952/jnva.4.2020.1.07

\title{
TENSOR VARIATIONAL INEQUALITIES: THEORETICAL RESULTS, NUMERICAL METHODS AND APPLICATIONS TO AN ECONOMIC EQUILIBRIUM MODEL
}

\author{
ANNAMARIA BARBAGALLO ${ }^{1, *}$, SERENA GUARINO LO BIANCO ${ }^{2}$, GERARDO TORALDO ${ }^{1}$ \\ ${ }^{1}$ Department of Mathematics and Applications "R. Caccioppoli”, \\ University of Naples Federico II, via Cintia - 80126 Naples, Italy \\ ${ }^{2}$ Department of Agriculture Science, University of Naples Federico II, via Università - 80055 Portici (NA), Italy
}

\begin{abstract}
The paper deals with the study of tensor variational inequalities. and some projection methods to solve them. In particular, some properties on the solutions to such an inequality are established and a fixed point theorem is proved. Moreover, some numerical methods are introduced and the convergence analysis of them is investigated. All the theoretical results are applied to analyze a general oligopolistic market equilibrium problem in which each firm produces several commodities and has some production excesses since the equilibrium condition is characterized by means of a tensor variational inequality. A numerical example is also discussed.
\end{abstract}

Keywords. Tensor variational inequality; Noncooperative game; Fixed point results; Numerical methods.

\section{INTRODUCTION}

In this paper, we analyze tensor variational inequalities and establish some useful properties in order to introduce some numerical algorithms for computing their solutions. We apply the obtained results to a general oligopolistic market equilibrium problem in presence of production excesses. Starting from the model in which firms produce several goods introduced in [2], it is reasonable that production excesses occur during some crisis periods or because of upper bounds in transportations (we assume that the firms and the demand markets are spatially separated and so it needs to ship commodities by means of limited transfer services). In particular, when the firms produce more quantity of commodities that they can send or the demand markets can buy, excesses need to be stored in appropriate storages. As a consequence, storage costs have to be considered in the model. Also for this extended model, the equilibrium condition is given by using a generalization of the Cournot-Nash principle. By using variational techniques, the equilibrium distribution can be characterized as the solution to a suitable tensor variational inequality. Thanks to this equivalence we can apply the theoretical results obtained

\footnotetext{
${ }^{*}$ Corresponding author.

E-mail addresses: annamaria.barbagallo@unina.it (A. Barbagallo), serena.guarinolobianco@unina.it (S. Guarino Lo Bianco), gerardo.toraldo@unina.it (G. Toraldo).

Received October 28, 2019; Accepted March 6, 2020.
}

(C)2020 Journal of Nonlinear and Variational Analysis 
for variational inequalities to the market model we consider. Moreover we prove some geometric properties on solutions to tensor variational inequalities and a fixed point theorem. By using this result, we are able to introduce some projection numerical methods for solving tensor variational inequalities. In particular, we present the extended version of projection method to tensor variational inequalities, and consider a more refined version called tensor extragradient method. Finally, inspired by the Marcotte adaptive steplenght algorithm, we introduce the tensor extragradient method with adaptive steplenght. For each numerical scheme, we investigate, under suitable assumptions, the convergence of iteratively generated sequences to solutions to tensor variational inequalities. Such inequalities were introduced, for the first time, in [2] and studied in [3]. Recently, several authors paid attention to variational inequalities involving tensors. For instance, a class of complementarity problems, called tensor complementarity problems were studied in [23]. Afterwards, Che, Qi and Wei studied the tensor complementarity problem and introduced a suitable tensor variational inequality which expresses the tensor complementarity problem for $X=\mathbb{R}_{+}^{n}$ (see [5]). For this reason in [27], some existence and uniqueness theorems were established for a special tensor variational inequality.

Tensor variational inequalities have also a natural application to the study of some economic problems. In particular, they have a fundamental role to analyze a general oligopolistic market equilibrium problem in which the firms produce several goods and compete with a noncooperative behavior. In 1897, Cournot in [6] examined, for the first time, the behavior between two producers of a given commodity which try, each one on his own, to maximize their respective profit. Nash, in $[19,20]$, extended Cournot's duopoly problem for a general model with $n$ agents, each acting according to his own self-interest, the so-called noncooperative game. Each player has at his disposal a strategy which he chooses from a set of feasible strategies with the aim of maximizing his utility level gives the decisions of the other players. Many scholars studied existence and uniqueness results for the noncooperative game under different assumptions (see, e.g., [11, 12, 13, 22]). Finally, we refer to [7] for the relationship between the oligopoly market model and the competitive spatial price equilibrium model established by means of the finite dimensional variational approach.

We underline that variational inequalities are a fundamental tool to study many problems arising from optimization theory and capture various applications, such as, partial differential equations, optimal control, and mathematical programming (see, e.g., [1, 10, 29]). For this reason, several authors introduced iterative methods for solving such inequalities, see, e.g., $[8,9,10,14,17,18,21,26,28]$. Two meaningful approaches for solving variational inequalities are the regularized method and the projection method. Starting from the simple gradient projection method, many different projection-like methods have been proposed to approximate solutions to variational inequality problems under various types of conditions. We cite, for instance, the extragradient methods and the subgradient extragradient methods. We emphasize that those methods use stepsizes which often depend on the Lipschitz constant or/and the modulus of strong monotonicity of the function considered and these constants are not always easy to obtain.

The paper is organized as follows. Section 2 concerns some preliminaries on tensor variational inequalities. In particular, we present some existence results and obtain a fixed point theorem which has a relevant role for the study of algorithms for solving tensor variational inequalities. In Section 3, some numerical methods based on the projection operator for solving 
tensor variational inequalities are introduced. Moreover, we prove that the algorithms generate a sequence of approximation solutions which converges to the tensor variational solution. Section 4 deals with the general oligopolistic market equilibrium problem in presence of production excesses. The equilibrium condition which generalizes the Cournot-Nash principle is presented and expressed by a suitable tensor variational inequality. Because of the variational formulation of the model, some existence and uniqueness results for the equilibrium distributions are shown. In Section 5, a numerical example is provided. Finally, Section 6 contains conclusive remarks.

\section{TENSOR VARIATIONAL INEQUALITIES}

Let $V$ be a finite-dimensional vector space endowed with an inner product denoted by $\langle\cdot, \cdot\rangle$. A $N$-order tensor is an element of the product space $V \times \cdots \times V$, i.e., a multidimensional array. Matrices, vectors and scalars are tensors of order two, one and zero, respectively. We adopt the following notations

- small letters $v, w, \ldots$, denote vectors;

- capital letters $A, B, \ldots$ denote matrices;

- italic capital letters $\mathscr{X}, \mathscr{Y}, \ldots$ denote tensors of general order.

A $N$-order tensor on a vector space $V$ of dimension $m$ has $m^{N}$ entries. Let us indicate the set of all the order $N$ tensors on the $m$-dimensional vector space $V$ with $\mathscr{T}_{N, m}(V)$. When the space $V$ is $\mathbb{R}^{m}$ or when the dimensions are clear in the context, we do not specify $V$ and the dimensions $N, m$ and we write only $\mathscr{T}_{N, m}$ and $\mathscr{T}$. The entries of a tensor $\mathscr{A}$ of order $N$ are real numbers indicated by $x_{i, j, k, \ldots,}$. The element $\left(i_{1}, i_{2}, \ldots, i_{N}\right)$ is denoted by $x_{i_{1}, i_{2}, \ldots, i_{N}}$.

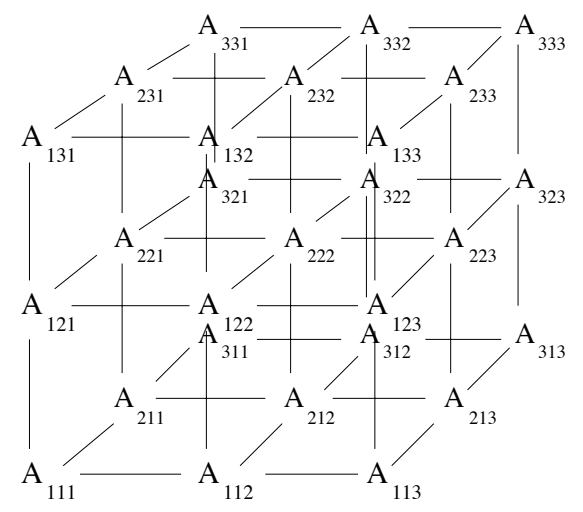

FIGURE 1. A 3-order tensor on a 3-dimensional vector space

We endow the vector space $\mathscr{T}_{N, m}$ with an inner product $\langle\cdot, \cdot\rangle$ as follows.

Definition 2.1. Let $\mathscr{X}, \mathscr{Y}$ be two tensors in $\mathscr{T}_{N, m}$. Let us define the mapping $\langle\cdot, \cdot\rangle: \mathscr{T}_{N, m} \times$ $\mathscr{T}_{N, m} \rightarrow \mathbb{R}$, indicate also with the notation ":", as

$$
\langle\mathscr{X}, \mathscr{Y}\rangle=\mathscr{X}: \mathscr{Y}=\sum_{i_{1}=1}^{m} \sum_{i_{2}=1}^{m} \cdots \sum_{i_{N}=1}^{m} x_{i_{1}, i_{2}, \ldots, i_{N}} y_{i_{1}, i_{2}, \ldots, i_{N}} .
$$


It is worth to remark that the previous mapping is an inner product on $\mathscr{T}_{N, m}$. Notice also that if $\mathscr{X}$ and $\mathscr{Y}$ are tensors of order two, i.e., matrices, the inner product defined above coincides with the usual one on the space of matrices, that is,

$$
\langle\mathscr{X}, \mathscr{Y}\rangle=\operatorname{tr}\left(\mathscr{X} \mathscr{Y}^{T}\right)
$$

where $\operatorname{tr}(\cdot)$ is the trace operator and ${ }^{T}$ denotes the transpose operation. Moreover, the norm $\|\cdot\|$ induced by $\langle\cdot, \cdot\rangle$ is the analogous one of the Frobenius norm for matrices. With this definition it is easy to prove that $\left(\mathscr{T}_{N, m},\langle\cdot, \cdot\rangle\right)$ is a Hilbert space.

A tensor variational inequality reads as follows.

Definition 2.2. Let $K$ be a nonempty closed convex subset of $\mathscr{T}_{N, m}$ and let $F: K \rightarrow \mathscr{T}_{N, m}$ be a tensor function. The tensor variational inequality is the problem of finding $\mathscr{X} \in K$ such that

$$
\langle F(\mathscr{X}), \mathscr{Y}-\mathscr{X}\rangle=F(\mathscr{X}):(\mathscr{Y}-\mathscr{X}) \geq 0, \quad \forall \mathscr{Y} \in K .
$$

We recall some existence and uniqueness results for tensor variational inequalities proved in [2] and [3]. In the bounded case of the set $K$, we state the following.

Theorem 2.1. Let $K$ be a nonempty compact convex subset of $\mathscr{T}_{N, m}$ and let $F: K \rightarrow \mathscr{T}_{N, m}$ be a continuous tensor function. Then tensor variational inequality problem (2.1) admits at least one solution.

From the previous result, it is easy to deduce that $\mathscr{X}$ is a solution to (2.1) if and only if $\mathscr{X}$ is a fixed point for $\mathscr{X} \mapsto \operatorname{Pr}_{K}(\mathscr{X}-\alpha F(\mathscr{X}))$, for any $\alpha>0$.

Without some boundness requirements on the set $K$, we can obtain an existence result supposing that of the operator $F$ is coercive, as the following result establishes.

Theorem 2.2. Let $K$ be a nonempty closed convex subset of $\mathscr{T}_{N, m}$ and let $F: K \rightarrow \mathscr{T}_{N, m}$ be a continuous tensor function satisfying the coercivity condition

$$
\lim _{\|\mathscr{X}\| \rightarrow+\infty} \frac{\left\langle F(\mathscr{X})-F\left(\mathscr{X}_{0}\right), \mathscr{X}-\mathscr{X}_{0}\right\rangle}{\left\|\mathscr{X}-\mathscr{X}_{0}\right\|}=+\infty,
$$

for some $\mathscr{X}_{0} \in K$. Then tensor variational inequality (2.1) admits a solution.

In the unbounded case, some existence and uniqueness results are based on monotone approach. For this reason, we recall some monotonicity conditions for tensor functions.

Definition 2.3. A tensor function $F: K \rightarrow \mathscr{T}_{N, m}$ is said to be

- monotone on $K$ if, for each $\mathscr{X}, \mathscr{Y} \in \mathscr{T}_{N, m}$,

$$
\langle F(\mathscr{X})-F(\mathscr{Y}), \mathscr{X}-\mathscr{Y}\rangle \geq 0 \text {; }
$$

- strictly monotone on $K$ if, for each $\mathscr{X}, \mathscr{Y} \in \mathscr{T}_{N, m}$, with $\mathscr{X} \neq \mathscr{Y}$,

$$
\langle F(\mathscr{X})-F(\mathscr{Y}), \mathscr{X}-\mathscr{Y}\rangle>0 \text {; }
$$

- strongly monotone on $K$ if, for each $\mathscr{X}, \mathscr{Y} \in \mathscr{T}_{N, m}$, there exists $v>0$ such that

$$
\langle F(\mathscr{X})-F(\mathscr{Y}), \mathscr{X}-\mathscr{Y}\rangle \geq v\|\mathscr{X}-\mathscr{Y}\|^{2} \text {. }
$$

Monotone assumptions ensure the existence of solutions to tensor variational inequalities, as below. 
Theorem 2.3. Let $K$ be a nonempty closed convex subset of $\mathscr{T}_{N, m}$ and let $F: K \rightarrow \mathscr{T}_{N, m}$ be a tensor function.

a) If $F$ is continuous and monotone, then the set of solutions $\operatorname{Sol}(F, K)$ to (2.1) is nonempty, closed and convex;

b) If $F$ is strictly monotone, then if there exists a solution to (2.1), then it is unique;

c) If $F$ is continuous and strongly monotone, then there exists a unique solution to (2.1).

By using Theorem 2.3, it is possible to obtain some interesting corollaries that we prove in the following.

Corollary 2.1. Let $K$ be a nonempty compact convex subset of $\mathscr{T}_{N, m}$, let $\mathscr{X}$ be a solution to (2.1) and suppose that $\mathscr{X} \in \stackrel{\circ}{K}$, where $\stackrel{\circ}{K}$ is the interior of $K$. Then $F(\mathscr{X})=0$.

Proof. Since $\mathscr{X} \in \stackrel{\circ}{K}$, we have that $\mathscr{Y}-\mathscr{X}$ describes a neighborhood of the origin. It means that, for any $\mathscr{Z} \in \mathscr{T}_{N, m}$, there exists $\varepsilon>0$ such that $\mathscr{Z}=\varepsilon(\mathscr{Y}-\mathscr{X})$. As a consequence, we have

$$
\langle F(\mathscr{X}), \mathscr{Z}\rangle=\varepsilon\langle F(\mathscr{X}), \mathscr{Y}-\mathscr{X}\rangle \geq 0, \quad \forall \mathscr{Y} \in \mathscr{T}_{N, m} .
$$

Then, it implies $F(\mathscr{X})=0$.

We introduce some other concepts.

Definition 2.4. Let $K$ be a convex subset of $\mathscr{T}_{N, m}$ and $\mathscr{X} \in \partial K$. A hyperplane

$$
\langle\mathscr{Z}, \mathscr{Y}-\mathscr{X}\rangle=0, \quad \mathscr{Z} \in \mathscr{T}_{N, m} \backslash\{0\},
$$

is said a support hyperplane of $K$ if

$$
\langle\mathscr{Z}, \mathscr{Y}-\mathscr{X}\rangle=0, \quad \forall \mathscr{Y} \in K .
$$

The following corollary holds.

Corollary 2.2. Let $\mathscr{X}$ be a solution to (2.1) and suppose that $\mathscr{X} \in \partial K$. Let $F: K \rightarrow \mathscr{T}_{N, m}$ be a tensor function. Then $F(\mathscr{X})$ determines a support hyperplane of $K$, provided $F(\mathscr{X}) \neq 0$.

Proof. The proof is immediate since the affine function $f(\mathscr{Y})=\langle F(\mathscr{X}), \mathscr{Y}-\mathscr{X}\rangle$ is nonnegative for $\mathscr{Y} \in \mathscr{T}_{N, m}$.

In order to obtain another corollary of Theorem 2.3, we need to present the following definition.

Definition 2.5. A tensor mapping $F: \mathscr{T}_{N, m} \rightarrow \mathscr{T}_{N, m}$ is a contraction mapping if

$$
\|F(\mathscr{X})-F(\mathscr{Y})\| \leq \alpha\|\mathscr{X}-\mathscr{Y}\|, \quad \forall \mathscr{X}, \mathscr{Y} \in \mathscr{T}_{N, m},
$$

for some $\alpha \in[0,1[$. If we allow $\alpha=1$, then $F$ is said to be nonexpansive.

Now, we are able to prove the next result.

Corollary 2.3. Let $K$ be a nonempty closed bounded subset of $\mathscr{T}_{N, m}$ and let $F: K \rightarrow K$ be a nonexpansive mapping. Then $F$ possess a nonempty closed convex subset $C \subset K$ of fixed points.

Proof. Since $F$ a nonexpansive mapping, we have that $I-F$ is monotone. Then, we may apply Theorem 2.3 to the tensor variational inequality associated to $I-F$. Since any solution to the tensor variational inequality for $I-F$ is a fixed point for $F$, the claim is achieved. 
The following result will be very useful to study the convergence analysis of the numerical methods that we will present in the next section.

Theorem 2.4. Let $K$ be a nonempty compact convex subset of $\mathscr{T}_{N, m}$ and let $F: K \rightarrow \mathscr{T}_{N, m}$ be a tensor continuous function. Then the function $F$ admits a fixed point in $K$.

Proof. Let $\Sigma$ be a closed ball contained the set $K$. The projection map $\operatorname{Pr}_{K}$ is continuous, so is $F \circ \operatorname{Pr}_{K}: \Sigma \rightarrow \Sigma$. By using Brower's fixed point theorem, there exists $\mathscr{X} \in \Sigma$ such that

$$
F \circ \operatorname{Pr}_{K}(\mathscr{X})=\mathscr{X} \text {. }
$$

Being $\operatorname{Pr}_{K}(\mathscr{X})=\mathscr{X}$, it results $F(\mathscr{X})=\mathscr{X}$. This completes the proof.

Let us finish the section with a useful Minty type preliminary result.

Lemma 2.1. Let $K$ be a nonempty closed convex subset of $\mathscr{T}_{N, m}$ and let $F: K \rightarrow \mathscr{T}_{N, m}$ be a tensor continuous and monotone function. The tensor variational ineqaulity (2.1) is equivalent to the Minty-Browder tensor variational inequality

$$
\langle F(\mathscr{Y}), \mathscr{Y}-\mathscr{X}\rangle \geq 0, \quad \forall \mathscr{Y} \in K .
$$

Proof. Theorem 2.3 ensures the existence of solutions to (2.1). Moreover, the monotonicity of $F$ and (2.1) imply, for any $\mathscr{Y} \in K$,

$$
\langle F(\mathscr{Y}), \mathscr{Y}-\mathscr{X}\rangle=\langle F(\mathscr{Y})-F(\mathscr{X}), \mathscr{Y}-\mathscr{X}\rangle+\langle F(\mathscr{X}), \mathscr{Y}-\mathscr{X}\rangle \geq 0 .
$$

In order to prove the converse, taking in (2.2)

$$
\mathscr{Y}=\mathscr{X}+\theta(\mathscr{Z}-\mathscr{X}) \in K,
$$

for $\theta \in] 0,1]$ and $\mathscr{Z} \in K$, it results

$$
\langle F(\mathscr{X}+\theta(\mathscr{Z}-\mathscr{X})), \mathscr{Y}-\mathscr{X}\rangle \geq 0 .
$$

We end up with (2.1) by letting $\theta \rightarrow 0$ and using the continuity of $F$.

\section{TENSOR NUMERICAL METHODS}

In this section, we analyze the structure of some algorithms for solving tensor variational inequalities as (2.1) based on the projection operator. More precisely, the numerical methods that we will present take into account of Fixed Point Theorem 2.4. Let us remind that Theorem 2.1 ensures that there exists a unique solution $\mathscr{X}^{*}$ to tensor variational inequality (2.1) provided that $K$ is a nonempty convex closed subset of $\mathscr{T}_{N, m}$ and $F$ is a tensor continuous and strongly monotone function on $K$. To the light of Theorem 2.3, it is reasonable to look for some numerical methods for computing the unique solution to tensor variational inequality (2.1).

3.1. Tensor projection method. The tensor projection method that we introduce here follows the procedure described below. Let $\mathscr{X}_{0} \in K$ be a fixed initial point and let $\alpha$ be a nonnegative number. Following the same spirit of the projection algorithm presented for vector variational inequalities in [24], we consider a numerical scheme that updates iteratively $\mathscr{X}_{k}$ according to the formula

$$
\mathscr{X}_{k+1}=\operatorname{Pr}_{K}\left(\mathscr{X}_{k}-\alpha F\left(\mathscr{X}_{k}\right)\right)
$$


where $\operatorname{Pr}_{K}(\cdot)$ denotes the orthogonal projection mapping onto $K$ and $\alpha$ will be suitable chosen. Observe that $\operatorname{Pr}_{K}\left(\mathscr{X}_{k}-\alpha F\left(\mathscr{X}_{k}\right)\right)$ is the solution of the following quadratic programming problem

$$
\min _{\mathscr{Y} \in K} \frac{1}{2}\langle\mathscr{Y}, \mathscr{Y}\rangle-\left\langle\mathscr{X}_{k}-\alpha F\left(\mathscr{X}_{k}\right), \mathscr{Y}\right\rangle
$$

for $k \in \mathbb{N}$. The key observation of the projection method is that $\mathscr{X}$ is a solution to (2.1) if and only if

$$
\mathscr{X}^{*}=\operatorname{Pr}_{K}\left(\mathscr{X}^{*}-\alpha F\left(\mathscr{X}^{*}\right)\right) .
$$

Indeed, we can use same arguments in the proof of Theorem 4.2 in [2] to obtain the previous equivalence.

The convergence analysis for this method relies on the contractive properties of operator $\mathscr{X} \mapsto \mathscr{X}-\alpha F(\mathscr{X})$ under the assumption that $F$ is strongly monotone and Lipschitz continuous. Indeed utilizing the non-expansiveness property of the projection operation, we can prove the following result.

Theorem 3.1. Let $X$ be a nonempty convex closed subset of $\mathscr{T}_{N, m}$. Let $F$ be a L-Lipschitz continuous and strongly monotone tensor function with constant $v$. If $\alpha \in\left(0,2 v / L^{2}\right)$, then the tensor projection method defined by (3.1) determines a sequence $\left\{\mathscr{X}_{k}\right\}$ converging to the solution to tensor variational inequality (2.1).

Proof. We first compute

$$
\begin{aligned}
& \left\|\operatorname{Pr}_{K}(\mathscr{X}-\alpha F(\mathscr{X}))-\operatorname{Pr}_{K}(\mathscr{Y}-\alpha F(\mathscr{Y}))\right\|^{2} \\
\leq & \|\mathscr{X}-\alpha F(\mathscr{X})-\mathscr{Y}-\alpha F(\mathscr{Y})\|^{2} \\
= & \left.\|\mathscr{X}-\mathscr{Y}\|^{2}-2 \alpha\langle F(\mathscr{X})-F(\mathscr{Y})), \mathscr{X}-\mathscr{Y}\right\rangle \\
& +\alpha^{2}\|F(\mathscr{X})-F(\mathscr{Y})\|^{2} \\
\leq & \left(1-2 \alpha v+\alpha^{2} L^{2}\right)\|\mathscr{X}-\mathscr{Y}\|^{2} .
\end{aligned}
$$

Letting $\alpha \in\left(0,2 v / L^{2}\right)$, we have that the projection operator is contractive.

Let us remark that the requirement that $F$ is strongly monotone is very strong, and often fails to be satisfied in applications; an execution of the algorithm requires $v$ as well as the Lipschitz constant $L$ and to estimate these parameters is in general non-trivial; a poor choice of these parameters can either lead to divergence or to a very slow convergence.

3.2. Tensor extragradient method. A more refined method for solving tensor variational inequalities is inspired by the algorithm considered in [4] and [25], that is, the tensor extragradient method. For a fixed initial point $\mathscr{X}_{0} \in K$ and $\alpha>0$, the algorithm updates iteratively $\mathscr{X}_{k}$ according to the double projection formula

$$
\begin{aligned}
& \overline{\mathscr{X}}_{k}=\operatorname{Pr}_{K}\left(\mathscr{X}_{k}-\alpha F\left(\mathscr{X}_{k}\right)\right), \\
& \mathscr{X}_{k+1}=\operatorname{Pr}_{K}\left(\mathscr{X}_{k}-\alpha F\left(\overline{\mathscr{X}}_{k}\right)\right) .
\end{aligned}
$$

It is possible to prove that the extragradient method determines a sequence $\left\{\mathscr{X}_{k}\right\}$ converging to the solution to (2.1) as the following result establishes. 
Theorem 3.2. Let $X$ be a nonempty convex closed subset of $\mathscr{T}_{N, m}$. Let $F$ be a L-Lipschitz continuous and monotone tensor function. If $\alpha \in(0,1 / L)$, then the extragradient method defined by (3.3) determines a sequence $\left\{\mathscr{X}_{k}\right\}$ converging to the solution to tensor variational inequality (2.1).

Proof. Firstly, we show that, for any solution $\mathscr{X}^{*}$ to $(2.1)$,

$$
\left\|\mathscr{X}_{k+1}-\mathscr{X}^{*}\right\|^{2} \leq\left\|\mathscr{X}_{k}-\mathscr{X}^{*}\right\|^{2}-\left(1-\alpha^{2} L^{2}\right)\left\|\mathscr{X}_{k}-\overline{\mathscr{X}}_{k}\right\|^{2} .
$$

Indeed, the monotonicity condition and the fact that $\mathscr{X}^{*}$ is a solution to (2.1) imply

$$
\begin{aligned}
0 \leq\left\langle F\left(\overline{\mathscr{X}}_{k}\right)-F\left(\mathscr{X}^{*}\right), \overline{\mathscr{X}}_{k}-\mathscr{X}^{*}\right\rangle & =\left\langle F\left(\overline{\mathscr{X}}_{k}\right), \overline{\mathscr{X}}_{k}-\mathscr{X}^{*}\right\rangle-\left\langle F\left(\mathscr{X}^{*}\right), \overline{\mathscr{X}}_{k}-\mathscr{X}^{*}\right\rangle \\
& \leq\left\langle F\left(\overline{\mathscr{X}}_{k}\right), \overline{\mathscr{X}}_{k}-\mathscr{X}^{*}\right\rangle \\
& =\left\langle F\left(\overline{\mathscr{X}}_{k}\right), \overline{\mathscr{X}}_{k}-\mathscr{X}_{k+1}\right\rangle \\
& +\left\langle F\left(\overline{\mathscr{X}}_{k}\right), \overline{\mathscr{X}}_{k+1}-\mathscr{X}^{*}\right\rangle .
\end{aligned}
$$

As a consequence, we obtain

$$
\left\langle F\left(\overline{\mathscr{X}}_{k}\right), \mathscr{X}^{*}-\overline{\mathscr{X}}_{k+1}\right\rangle \leq\left\langle F\left(\overline{\mathscr{X}}_{k}\right), \overline{\mathscr{X}}_{k}-\mathscr{X}_{k+1}\right\rangle .
$$

By the definition of $\mathscr{X}_{k+1}$ and using the previous inequality, we deduce

$$
\begin{aligned}
\left\|\mathscr{X}_{k+1}-\mathscr{X}^{*}\right\|^{2} \leq & \left\|\mathscr{X}_{k}-\alpha F\left(\overline{\mathscr{X}}_{k}\right)-\mathscr{X}^{*}\right\|^{2}-\left\|\mathscr{X}_{k}-\alpha F\left(\overline{\mathscr{X}}_{k}\right)-\mathscr{X}_{k+1}\right\|^{2} \\
= & \left\|\mathscr{X}_{k}-\mathscr{X}^{*}\right\|^{2}-\left\|\mathscr{X}_{k}-\mathscr{X}_{k+1}\right\|^{2}+2 \alpha\left\langle F\left(\overline{\mathscr{X}}_{k}\right), \mathscr{X}^{*}-\mathscr{X}_{k+1}\right\rangle \\
\leq & \left\|\mathscr{X}_{k}-\mathscr{X}^{*}\right\|^{2}-\left\|\mathscr{X}_{k}-\overline{\mathscr{X}}_{k}\right\|^{2}-\left\|\overline{\mathscr{X}}_{k}-\mathscr{X}_{k+1}\right\|^{2} \\
& -2\left\langle\left(\mathscr{X}_{k}-\overline{\mathscr{X}}_{k}\right), \overline{\mathscr{X}}_{k}-\mathscr{X}_{k+1}\right\rangle+2 \alpha\left\langle F\left(\overline{\mathscr{X}}_{k}\right), \overline{\mathscr{X}}_{k}-\mathscr{X}_{k+1}\right\rangle .
\end{aligned}
$$

Hence, we have

$$
\begin{aligned}
\left\|\mathscr{X}_{k+1}-\mathscr{X}^{*}\right\|^{2} \leq & \left\|\mathscr{X}_{k}-\mathscr{X}^{*}\right\|^{2}-\left\|\mathscr{X}_{k}-\overline{\mathscr{X}}_{k}\right\|^{2}-\left\|\overline{\mathscr{X}}_{k}-\mathscr{X}_{k+1}\right\|^{2} \\
& +2\left\langle\mathscr{X}_{k+1}-\overline{\mathscr{X}}_{k}, \mathscr{X}_{k}-\alpha F\left(\overline{\mathscr{X}}_{k}\right)-\overline{\mathscr{X}}_{k}\right\rangle
\end{aligned}
$$

By the definition of $\overline{\mathscr{X}}_{k}$ and using the $L$-Lipschitz continuity of $F$, it results

$$
\begin{aligned}
&\left\langle\mathscr{X}_{k+1}-\overline{\mathscr{X}}_{k}, \mathscr{X}_{k}-\alpha F\left(\overline{\mathscr{X}}_{k}\right)-\overline{\mathscr{X}}_{k}\right\rangle=\left\langle\mathscr{X}_{k+1}-\overline{\mathscr{X}}_{k}, \mathscr{X}_{k}-\alpha F\left(\mathscr{X}_{k}\right)-\overline{\mathscr{X}}_{k}\right\rangle \\
&-\alpha\left\langle\mathscr{X}_{k+1}-\overline{\mathscr{X}}_{k}, F\left(\mathscr{X}_{k}\right)-F\left(\overline{\mathscr{X}}_{k}\right)\right\rangle \\
& \leq \alpha\left\langle\mathscr{X}_{k+1}-\overline{\mathscr{X}}_{k}, F\left(\mathscr{X}_{k}\right)-F\left(\overline{\mathscr{X}}_{k}\right)\right\rangle \\
& \leq \alpha L\left\|\mathscr{X}_{k+1}-\overline{\mathscr{X}}_{k}\right\| \cdot\left\|\mathscr{X}_{k}-\overline{\mathscr{X}}_{k}\right\| .
\end{aligned}
$$

Taking into account (3.4) and (3.5), we deduce

$$
\begin{aligned}
\left\|\mathscr{X}_{k+1}-\mathscr{X}^{*}\right\|^{2} \leq & \left\|\mathscr{X}_{k}-\mathscr{X}^{*}\right\|^{2}-\left\|\mathscr{X}_{k}-\overline{\mathscr{X}}_{k}\right\|^{2}-\left\|\bar{X}_{k}-\mathscr{X}_{k+1}\right\|^{2} \\
& -\alpha L\left\|\mathscr{X}_{k+1}-\overline{\mathscr{X}}_{k}\right\| \cdot\left\|\mathscr{X}_{k}-\overline{\mathscr{X}}_{k}\right\| . \\
= & \left\|\mathscr{X}_{k}-\mathscr{X}^{*}\right\|^{2}-\left(1-\alpha^{2} L^{2}\right)\left\|\mathscr{X}_{k}-\overline{\mathscr{X}}_{k}\right\|^{2} \\
& -\left(\alpha L\left\|\mathscr{X}_{k+1}-\overline{\mathscr{X}}_{k}\right\|-\left\|\overline{\mathscr{X}}_{k}-\mathscr{X}_{k+1}\right\|^{2}\right)^{2} \\
\leq & \left\|\mathscr{X}_{k}-\mathscr{X}^{*}\right\|^{2}-\left(1-\alpha^{2} L^{2}\right)\left\|\mathscr{X}_{k}-\overline{\mathscr{X}}_{k}\right\|^{2} .
\end{aligned}
$$

Then, for $\alpha \in(0,1 / L)$, the method converges to the solution to (2.1). 
It is worth to point out that the tensor extragradient method converges faster than the tensor projection method, even if the convergence of these methods is ensured under similar assumptions.

3.3. Tensor extragradient method with adaptive steplenght. We introduce now an algorithm for solving tensor variational inequality (2.1) making use of a modified version of the tensor extragradient method inspired by the scheme considered by Marcotte in [15].

The algorithm starting from an initial tensor $\mathscr{X}_{0} \in K$ and a fixed number $\alpha_{0}>0$, iteratively updates $\mathscr{X}_{k+1}$ from $\mathscr{X}_{k}$ according to the following projection formulas:

$$
\begin{aligned}
& \tilde{\mathscr{X}}_{k}=\operatorname{Pr}_{K}\left(\mathscr{X}_{k}-\alpha_{k} F\left(\mathscr{X}_{k}\right)\right), \\
& \mathscr{X}_{k+1}=\operatorname{Pr}_{K}\left(\mathscr{X}_{k}-\alpha_{k} F\left(\tilde{\mathscr{X}}_{k}\right)\right),
\end{aligned}
$$

for $k \in \mathbb{N}$, where $\alpha_{k}$ is chosen as following:

$$
\alpha_{k}=\min \left\{\frac{\alpha_{k-1}}{2}, \frac{\left\|\mathscr{X}_{k}-\tilde{\mathscr{X}}_{k}\right\|}{\sqrt{2}\left\|F\left(\mathscr{X}_{k}\right)-F\left(\tilde{\mathscr{X}}_{k}\right)\right\|}\right\} \text {. }
$$

We show that the tensor extragradient method with adaptive steplenght determines a sequence $\left\{\mathscr{X}_{k}\right\}$ converging to the solution to (2.1) under suitable assumptions. To this aim, as preliminary step, we prove the following Lemma.

Lemma 3.1. Let $X$ be a nonempty convex closed subset of $\mathscr{T}_{N, m}$. Let $F$ be a L-Lipschitz continuous and monotone tensor function. Let $\mathscr{X}_{k}$ be the sequence defined by (3.6). Then, for any nonnegative sequence $\left\{\alpha_{k}\right\}_{k}$,

$$
\left\|\mathscr{X}_{k+1}-\mathscr{X}^{*}\right\|^{2} \leq\left\|\mathscr{X}_{k}-\mathscr{X}^{*}\right\|^{2}-\left\|\mathscr{X}_{k}-\tilde{\mathscr{X}}_{k}\right\|^{2}\left(1-\alpha_{k}^{2} \frac{\left\|F\left(\mathscr{X}_{k}\right)-F\left(\tilde{\mathscr{X}}_{k}\right)\right\|^{2}}{\left\|\mathscr{X}_{k}-\tilde{\mathscr{X}}_{k}\right\|^{2}}\right) .
$$

Proof. Given two tensors $\mathscr{A}$ and $\mathscr{B}$, it is simple to obtain that

$$
\|\mathscr{A}-\mathscr{B}\|^{2} \geq\left\|\mathscr{A}-P_{K}(\mathscr{A})\right\|^{2}+\left\|\mathscr{B}-P_{K}(\mathscr{A})\right\|^{2}
$$

since

$$
\left\langle\mathscr{A}-P_{K}(\mathscr{A}), \mathscr{B}-P_{K}(\mathscr{A})\right\rangle \leq 0 \quad \forall \mathscr{B} \in K, \forall \mathscr{A} \in \mathscr{T}_{N, m} .
$$

Setting $\mathscr{A}=\mathscr{X}_{k}-\alpha_{k} F\left(\tilde{\mathscr{X}}_{k}\right), \mathscr{B}=\mathscr{X}^{*}$ and $\mathscr{X}_{k+1}=P_{K}(\mathscr{A})$ in (3.7), rearranging terms, we obtain

$$
\begin{aligned}
\left\|\mathscr{X}_{k+1}-\mathscr{X}^{*}\right\|^{2} & \leq\left\|\mathscr{X}_{k}-\alpha_{k} F\left(\tilde{\mathscr{X}}_{k}\right)-\mathscr{X}^{*}\right\|^{2}-\left\|\mathscr{X}_{k}-\alpha_{k} F\left(\tilde{\mathscr{X}}_{k}\right)-\mathscr{X}_{k+1}\right\|^{2} \\
& =\left\|\mathscr{X}_{k}-\mathscr{X}^{*}\right\|^{2}-\left\|\mathscr{X}_{k}-\mathscr{X}_{k+1}\right\|^{2}+2 \alpha_{k}\left\langle\mathscr{X}^{*}-\mathscr{X}_{k+1}, F\left(\tilde{\mathscr{X}}_{k}\right)\right\rangle .
\end{aligned}
$$

Using Lemma 2.1, we have

$$
\begin{aligned}
\left\langle\mathscr{X}^{*}-\mathscr{X}_{k+1}, F\left(\tilde{\mathscr{X}}_{k}\right)\right\rangle & =\left\langle\mathscr{X}^{*}-\tilde{\mathscr{X}}_{k}, F\left(\tilde{\mathscr{X}}_{k}\right)\right\rangle+\left\langle\tilde{\mathscr{X}}_{k}-\mathscr{X}_{k+1}, F\left(\tilde{\mathscr{X}}_{k}\right)\right\rangle \\
& \leq\left\langle\tilde{\mathscr{X}}_{k}-\mathscr{\mathscr { X }}_{k+1}, F\left(\tilde{\mathscr{X}}_{k}\right)\right\rangle .
\end{aligned}
$$


Using (3.9) and (3.10) and (3.8), we have

$$
\begin{aligned}
\| & \mathscr{X}_{k+1}-\mathscr{X}^{*} \|^{2} \\
\leq & \left\|\mathscr{X}_{k}-\mathscr{X}^{*}\right\|^{2}-\left\|\mathscr{X}_{k}-\tilde{\mathscr{X}}_{k}\right\|^{2}-\left\|\tilde{\mathscr{X}}_{k}-\mathscr{X}_{k+1}\right\|^{2} \\
& -2\left\langle\mathscr{X}_{k}-\tilde{\mathscr{X}}_{k}, \tilde{\mathscr{X}}_{k}-\mathscr{X}_{k+1}\right\rangle+2 \alpha_{k}\left\langle\tilde{\mathscr{X}}_{k}-\mathscr{X}_{k+1}, F\left(\tilde{\mathscr{X}}_{k}\right)\right\rangle \\
\leq & \left\|\mathscr{X}_{k}-\mathscr{X}^{*}\right\|^{2}-\left\|\mathscr{X}_{k}-\tilde{\mathscr{X}}_{k}\right\|^{2}-\left\|\tilde{\mathscr{X}}_{k}-\mathscr{X}_{k+1}\right\|^{2} \\
& +2\left\langle\mathscr{X}_{k}-\alpha_{k} F\left(\tilde{\mathscr{X}}_{k}\right)-\tilde{\mathscr{X}}_{k}, \mathscr{X}_{k+1}-\tilde{\mathscr{X}}_{k}\right\rangle \\
= & \left\|\mathscr{X}_{k}-\mathscr{X}^{*}\right\|^{2}-\left\|\mathscr{X}_{k}-\tilde{\mathscr{X}}_{k}\right\|^{2}-\left\|\tilde{\mathscr{X}}_{k}-\mathscr{X}_{k+1}\right\|^{2} \\
& +2\left\langle\mathscr{X}_{k}-\alpha_{k} F\left(\mathscr{X}_{k}\right)-\tilde{\mathscr{X}}_{k}, \mathscr{X}_{k+1}-\tilde{\mathscr{X}}_{k}\right\rangle+2 \alpha_{k}\left\langle F\left(\mathscr{X}_{k}\right)-F\left(\tilde{\mathscr{X}}_{k}\right), \mathscr{X}_{k+1}-\tilde{\mathscr{X}}_{k}\right\rangle \\
\leq & \left\|\mathscr{X}_{k}-\mathscr{X}^{*}\right\|^{2}-\left\|\mathscr{X}_{k}-\tilde{\mathscr{X}}_{k}\right\|^{2}-\left\|\tilde{\mathscr{X}}_{k}-\mathscr{X}_{k+1}\right\|^{2} \\
& +2 \alpha_{k}\left\|F\left(\mathscr{X}_{k}\right)-F\left(\tilde{\mathscr{X}}_{k}\right)\right\| \cdot\left\|\mathscr{X}_{k+1}-\tilde{\mathscr{X}}_{k}\right\| .
\end{aligned}
$$

Using the inequality

$$
\left\|\mathscr{X}_{k+1}-\tilde{\mathscr{X}}_{k}\right\|^{2}+\alpha_{k}^{2}\left\|F\left(\mathscr{X}_{k}\right)-F\left(\tilde{\mathscr{X}}_{k}\right)\right\|^{2} \geq 2 \alpha_{k}\left\|F\left(\mathscr{X}_{k}\right)-F\left(\tilde{\mathscr{X}}_{k}\right)\right\| \cdot\left\|\mathscr{X}_{k+1}-\tilde{\mathscr{X}}_{k}\right\|
$$

in (3.11), we obtain

$$
\begin{aligned}
\left\|\mathscr{X}_{k+1}-\mathscr{X}^{*}\right\|^{2} \leq & \left\|\mathscr{X}_{k}-\mathscr{X}^{*}\right\|^{2}-\left\|\mathscr{X}_{k}-\tilde{\mathscr{X}}_{k}\right\|^{2}-\left\|\tilde{\mathscr{X}}_{k}-\mathscr{X}_{k+1}\right\|^{2} \\
& +\left\|\mathscr{X}_{k+1}-\tilde{\mathscr{X}}_{k}\right\|^{2}+\alpha_{k}^{2}\left\|F\left(\mathscr{X}_{k}\right)-F\left(\tilde{\mathscr{X}}_{k}\right)\right\|^{2} \\
\leq & \left\|\mathscr{X}_{k}-\mathscr{X}^{*}\right\|^{2}-\left\|\mathscr{X}_{k}-\tilde{\mathscr{X}}_{k}\right\|^{2}\left(1-\alpha_{k}^{2} \frac{\left\|F\left(\mathscr{X}_{k}\right)-F\left(\tilde{\mathscr{X}}_{k}\right)\right\|^{2}}{\left\|\mathscr{X}_{k}-\tilde{\mathscr{X}}_{k}\right\|^{2}}\right) .
\end{aligned}
$$

Now we are able to obtain the convergence of the tensor extragradient method with adaptive steplenght.

Theorem 3.3. Let $X$ be a nonempty closed convex subset of $\mathscr{T}_{N, m}$. Let $F$ be a L-Lipschitz continuous and strongly monotone tensor function. Then the tensor extragradient method with adaptive steplenght defined by (3.6) determines a sequence $\left\{\mathscr{X}_{k}\right\}$ converging to the solution to tensor variational inequality (2.1).

Proof. We have to prove more precisely that there exists an index $M$ such that, for all $k>M$,

$$
\left\|\mathscr{X}_{k+1}-\mathscr{X}^{*}\right\|^{2} \leq \frac{\beta^{2}+\alpha^{2} L^{2}}{\beta^{2}+1}\left\|\mathscr{X}_{k}-\mathscr{X}^{*}\right\|^{2},
$$

where $\alpha$ is any positive constant satisfying $0<\alpha<\liminf \alpha_{k}$ and $\beta=(L+1 / \alpha) / v$.

It results from Lemma 3.1 that

$$
\begin{aligned}
\left\|\mathscr{X}_{k+1}-\mathscr{X}^{*}\right\|^{2} & \leq\left\|\mathscr{X}_{k}-\mathscr{X}^{*}\right\|^{2}-\left\|\mathscr{X}_{k}-\tilde{\mathscr{X}}_{k}\right\|^{2}\left(1-\alpha_{k}^{2} \frac{\left\|F\left(\mathscr{X}_{k}\right)-F\left(\tilde{\mathscr{X}}_{k}\right)\right\|^{2}}{\left\|\mathscr{X}_{k}-\tilde{\mathscr{X}}_{k}\right\|^{2}}\right) \\
& \leq\left\|\mathscr{X}_{k}-\mathscr{X}^{*}\right\|^{2}-\left\|\mathscr{X}_{k}-\tilde{\mathscr{X}}_{k}\right\|^{2}\left(1-\alpha_{k}^{2} L^{2}\right) \\
& =\left\|\mathscr{X}_{k}-\mathscr{X}^{*}\right\|^{2}\left(1-\left(1-\alpha_{k}^{2} L^{2}\right) \frac{\left\|\mathscr{X}_{k}-\tilde{\mathscr{X}}_{k}\right\|^{2}}{\left\|\mathscr{X}_{k}-\mathscr{X}^{*}\right\|^{2}}\right) .
\end{aligned}
$$


By definition of $\tilde{\mathscr{X}}_{k}$, it results

$$
\left\langle\tilde{\mathscr{X}}_{k}-\mathscr{Y}, \tilde{\mathscr{X}}_{k}-\mathscr{X}_{k}+\alpha_{k} F\left(\mathscr{X}_{k}\right)\right\rangle \leq 0, \quad \forall \mathscr{Y} \in K,
$$

or equivalently,

$$
\left\langle\tilde{\mathscr{X}}_{k}-\mathscr{Y}, \tilde{\mathscr{X}}_{k}-\mathscr{X}_{k}+\alpha_{k}\left[F\left(\mathscr{X}_{k}\right)-F\left(\tilde{\mathscr{X}}_{k}\right)\right]+\alpha_{k} F\left(\tilde{\mathscr{X}}_{k}\right)\right\rangle \leq 0, \quad \forall \mathscr{Y} \in K .
$$

Then, by using the Cauchy-Schwarz inequality and since $F$ is a $L$-Lipschitz continuous mapping, it follows

$$
\begin{aligned}
\alpha_{k}\left\langle\tilde{\mathscr{X}}_{k}-\mathscr{Y}, F\left(\tilde{\mathscr{X}}_{k}\right)\right\rangle & \leq-\left\langle\tilde{\mathscr{X}}_{k}-\mathscr{Y}, \tilde{\mathscr{X}}_{k}-\mathscr{X}_{k}\right\rangle+\alpha_{k}\left\langle\tilde{\mathscr{X}}_{k}-\mathscr{Y}, F\left(\tilde{\mathscr{X}}_{k}\right)-F\left(\mathscr{X}_{k}\right)\right\rangle \\
& \leq\left(1+\alpha_{k} L\right)\left\|\tilde{\mathscr{X}}_{k}-\mathscr{Y}\right\| \cdot\left\|\tilde{\mathscr{X}}_{k}-\mathscr{X}_{k}\right\|, \quad \forall \mathscr{Y} \in K .
\end{aligned}
$$

Hence,

$$
\left\langle\tilde{\mathscr{X}}_{k}-\mathscr{Y}, F\left(\tilde{\mathscr{X}}_{k}\right)\right\rangle \leq\left(L+1 / \alpha_{k}\right)\left\|\tilde{\mathscr{X}}_{k}-\mathscr{Y}\right\| \cdot\left\|\tilde{\mathscr{X}}_{k}-\mathscr{X}_{k}\right\|, \quad \forall \mathscr{Y} \in K .
$$

Setting $\mathscr{Y}=\mathscr{X}^{*}$ in the previous inequality, we obtain

$$
\left\langle\tilde{\mathscr{X}}_{k}-\mathscr{X}^{*}, F\left(\tilde{\mathscr{X}}_{k}\right)\right\rangle \leq\left(L+1 / \alpha_{k}\right)\left\|\tilde{\mathscr{X}}_{k}-\mathscr{X}^{*}\right\| \cdot\left\|\tilde{\mathscr{X}}_{k}-\mathscr{X}_{k}\right\| .
$$

It results

$$
\begin{aligned}
\left\langle\tilde{\mathscr{X}}_{k}-\mathscr{X}^{*}, F\left(\tilde{\mathscr{X}}_{k}\right)-F\left(\mathscr{X}^{*}\right)\right\rangle & =\left\langle\tilde{\mathscr{X}}_{k}-\mathscr{X}^{*}, F\left(\tilde{\mathscr{X}}_{k}\right)\right\rangle-\left\langle\tilde{\mathscr{X}}_{k}-\mathscr{X}^{*}, F\left(\mathscr{X}^{*}\right)\right\rangle \\
& \leq\left\langle\tilde{\mathscr{X}}_{k}-\mathscr{X}^{*}, F\left(\tilde{\mathscr{X}}_{k}\right)\right\rangle \\
& \leq\left(L+1 / \alpha_{k}\right)\left\|\tilde{\mathscr{X}}_{k}-\mathscr{X}^{*}\right\| \cdot\left\|\tilde{\mathscr{X}}_{k}-\mathscr{X}_{k}\right\| .
\end{aligned}
$$

Therefore, making use of the strongly monotonicity of $F$, we deduce

$$
v\left\|\tilde{\mathscr{X}}_{k}-\mathscr{X}^{*}\right\|^{2} \leq\left(L+1 / \alpha_{k}\right)\left\|\tilde{\mathscr{X}}_{k}-\mathscr{X}^{*}\right\| \cdot\left\|\tilde{\mathscr{X}}_{k}-\mathscr{X}_{k}\right\| .
$$

As a consequence, we have

$$
\begin{aligned}
\left\|\tilde{\mathscr{X}}_{k}-\mathscr{X}^{*}\right\| & \leq \frac{1}{v}\left(L+\frac{1}{\alpha}\right)\left\|\tilde{\mathscr{X}}_{k}-\mathscr{X}_{k}\right\| \\
& =\beta\left\|\tilde{\mathscr{X}}_{k}-\mathscr{X}_{k}\right\|, \quad \forall k>M,
\end{aligned}
$$

where $\beta=\frac{1}{v}\left(L+\frac{1}{\alpha}\right)$. Taking into account (3.14), we get

$$
\begin{aligned}
\left\|\mathscr{X}_{k}-\mathscr{X}^{*}\right\|^{2} & \leq\left\|\mathscr{X}_{k}-\tilde{\mathscr{X}}_{k}\right\|^{2}+\left\|\tilde{\mathscr{X}}_{k}-\mathscr{X}^{*}\right\|^{2} \\
& \leq\left(1+\beta^{2}\right)\left\|\mathscr{X}_{k}-\tilde{\mathscr{X}}_{k}\right\|^{2},
\end{aligned}
$$

which implies

$$
\frac{\left\|\mathscr{X}_{k}-\tilde{\mathscr{X}}_{k}\right\|^{2}}{\left\|\mathscr{X}_{k}-\mathscr{X}^{*}\right\|^{2}} \geq \frac{1}{1+\beta^{2}} .
$$

By using (3.13) and (3.14), we conclude

$$
\begin{aligned}
\left\|\mathscr{X}_{k+1}-\mathscr{X}^{*}\right\|^{2} & \leq\left(1-\frac{1-\alpha^{2} L^{2}}{1+\beta^{2}}\right)\left\|\mathscr{X}_{k}-\mathscr{X}^{*}\right\|^{2} \\
& =\frac{\beta^{2}+\alpha^{2} L^{2}}{\beta^{2}+1}\left\|\mathscr{X}_{k}-\mathscr{X}^{*}\right\|^{2} .
\end{aligned}
$$

This completes the proof. 


\section{AN OLIGOPOLISTIC MARKET MODEL}

We present a more general market equilibrium model with respect to the one introduced in [2]. Let us consider $m$ firms $P_{i}, i=1, \ldots, m$, and $n$ demand markets $Q_{j}, j=1, \ldots, n$, that are generally spatially separated. We assume that every firm $P_{i}$ produces a certain number $l$ of different commodities. Furthermore the commodities of kind $k=1, \ldots, l$, produced by the firm $P_{i}$, are consumed by markets $Q_{j}, j=1, \ldots, n$. We fix the following notations:

- $x_{i j}^{k}, i=1, \ldots, m, j=1, \ldots, n, k=1, \ldots, l$, is the nonnegative variable expressing the commodity shipment of kind $k$ between the producer $P_{i}$ and the market $Q_{j}$;

- $\varepsilon_{i}^{k}, i=1, \ldots, m, k=1, \ldots, l$, is the nonnegative variable expressing the production excess for the commodity of kind $k$ produced by firm $P_{i}$.

We suppose that both variables $x_{i j}^{k}$ and $\varepsilon_{i}^{k}$ are nonnegative and the following capacity contraints hold:

$$
\underline{x}_{i j}^{k} \leq x_{i j}^{k} \leq \bar{x}_{i j}^{k}, \quad \forall i=1, \ldots, m, \forall j=1, \ldots, n, \forall k=1, \ldots, l,
$$

where $\underline{x}_{i j}^{k}, \bar{x}_{i j}^{k}$ are nonnegative bounds. We observe that $x=\left(x_{i j}^{k}\right)_{i j}^{k} \in \mathscr{T}\left(\mathbb{R}_{+}\right)$, as well as, $\underline{x}=$ $\left(\underline{x}_{i j}^{k}\right)_{i j}^{k}$ and $\bar{x}=\left(\bar{x}_{i j}^{k}\right)_{i j}^{k}$. Moreover, we define the following quantities associated to the firm $P_{i}$, $i=1, \ldots, m$, and the demand market $Q_{j}, j=1, \ldots, n$ :

- $p_{i}^{k}, i=1, \ldots, m, k=1, \ldots, l$, is the variable expressing the commodity output of kind $k$ produced by $P_{i}, i=1, \ldots, m$;

- $q_{j}^{k}, j=1, \ldots, n$, is the variable expressing the demand for the commodity of kind $k$ of demand market $Q_{j}, j=1, \ldots, n$.

We assume that both variables $p_{i}^{k}$ and $q_{j}^{k}$ are nonnegative and the following feasibility conditions holds:

$$
p_{i}^{k}=\sum_{j=1}^{n} x_{i j}^{k}+\varepsilon_{i}^{k}, \quad \forall i=1, \ldots, m, \forall k=1, \ldots, l .
$$

The above conditions mean that the quantity produced by each firm $P_{i}$ of kind $k$ must be equal to the commodity shipments of such kind from that firm to all the demand markets plus the production excess for such kind of commodity. Summing up all the different kinds of commodities, the firm $P_{i}$ produces in total a quantity $p_{i}$ that can be expressed as

$$
p_{i}=\sum_{k=1}^{l}\left[\sum_{j=1}^{n} x_{i j}^{k}+\varepsilon_{i}^{k}\right], \quad \forall i=1, \ldots, m .
$$

Hence, the feasible set is given by

$$
\begin{aligned}
\widetilde{\mathbb{K}}=\left\{(x, \varepsilon) \in \mathscr{T}\left(\mathbb{R}_{+}\right) \times \mathbb{R}^{m l}:\right. \\
\underline{x}_{i j}^{k} \leq x_{i j}^{k} \leq \bar{x}_{i j}^{k}, \quad \forall i=1, \ldots, m, \forall j=1, \ldots, n, \forall k=1, \ldots, l, \\
\left.p_{i}^{k}=\sum_{j=1}^{n} x_{i j}^{k}+\varepsilon_{i}^{k}, \quad \forall i=1, \ldots, m, \forall k=1, \ldots, l\right\} .
\end{aligned}
$$

Let us note that $\widetilde{\mathbb{K}}$ is a nonempty convex compact subset of the Hilbert space $\mathscr{T}\left(\mathbb{R}_{+}\right)$.

We define now some quantities related to costs and prices that appear in the model. More precisely: 
- $\widetilde{f}_{i}^{k}, i=1, \ldots, m, k=1, \ldots, l$ denotes the variable expressing the production cost of $P_{i}$ for each good of type $k$. We assume that the production cost of a firm $P_{i}$ may depend upon the entire production pattern, namely, $\widetilde{f}_{i}^{k}=\widetilde{f}_{i}^{k}(x, \varepsilon), x \in \mathscr{T}\left(\mathbb{R}_{+}\right), \varepsilon \in \mathbb{R}^{m l}$;

- $\widetilde{d}_{j}^{k}, j=1, \ldots, n, k=1, \ldots, l$, denotes the variable expressing the demand price for unity of kind $k$ of the commodity with each demand market $Q_{j}$. We assume that the demand price of a demand market $Q_{j}$ may depend upon the entire consumption pattern, namely, $\widetilde{d}_{j}^{k}=\widetilde{d}_{j}^{k}(x, \varepsilon), x \in \mathscr{T}\left(\mathbb{R}_{+}\right), \varepsilon \in \mathbb{R}^{m p}$

- $\widetilde{c}_{i j}^{k}, i=1, \ldots, m, j=1, \ldots, n, k=1, \ldots, l$, denotes the variable expressing the transaction cost between firm $P_{i}$ and demand market $Q_{j}$ regarding the good of kind $k$. We assume also that the transaction cost depends upon the entire shipment pattern, namely, $\widetilde{c}_{i j}^{k}=$ $\widetilde{c}_{i j}^{k}(x, \varepsilon), x \in \mathscr{T}\left(\mathbb{R}_{+}\right), \varepsilon \in \mathbb{R}^{m l}$.

Since production excesses occur, we consider also the variable $\tilde{g}_{i}^{k}, i=1, \ldots, m, k=1, \ldots, l$, expressing the storage cost of the commodity of kind $k$ produced by the firm $P_{i}$ and assume that this cost may depend upon the entire production pattern, namely, $\widetilde{g}_{i}^{k}=\widetilde{g}_{i}^{k}(x, \varepsilon), x \in \mathscr{T}\left(\mathbb{R}_{+}\right)$, $\varepsilon \in \mathbb{R}^{m l}$.

Taking into account the above notations, the profit $v_{i}$ of the firm $P_{i}, i=1, \ldots, m$, is given by

$$
\widetilde{v}_{i}(x, \varepsilon)=\sum_{k=1}^{l}\left[\sum_{j=1}^{n} \widetilde{d}_{j}^{k}(x, \varepsilon) x_{i j}^{k}-\widetilde{f}_{i}^{k}(x, \varepsilon)-\widetilde{g}_{i}^{k}(x, \varepsilon)-\sum_{j=1}^{n} \widetilde{c}_{i j}^{k}(x, \varepsilon) x_{i j}^{k}\right],
$$

i.e., the difference between the price that each demand market $P_{i}$ is disposed to pay, the storage cost and the sum of the production costs and the transportation costs.

Let us observe that, by (4.1), we can express the production excesses in the following way

$$
\varepsilon_{i}^{k}=p_{i}^{k}-\sum_{j=1}^{n} x_{i j}^{k}, \quad \forall i=1, \ldots, m, \forall k=1, \ldots, l .
$$

Consequently, we have

$$
\sum_{k=1}^{l} \varepsilon_{i}^{k}=p_{i}-\sum_{k=1}^{l} \sum_{j=1}^{n} x_{i j}^{k}, \quad \forall i=1, \ldots, m .
$$

Using the nonnegativity of the production excesses, we can write an equivalent expression for the feasible set

$$
\begin{aligned}
\mathbb{K}= & \left\{x \in \mathscr{T}\left(\mathbb{R}_{+}\right):\right. \\
& \underline{x}_{i j}^{k} \leq x_{i j}^{k} \leq \bar{x}_{i j}^{k}, \quad \forall i=1, \ldots, m, \forall j=1, \ldots, n, \forall k=1, \ldots, l, \\
& \left.\sum_{j=1}^{n} x_{i j}^{k} \leq p_{i}^{k}, \quad \forall i=1, \ldots, m, \forall k=1, \ldots, l\right\} .
\end{aligned}
$$

It is worth to underline that $\mathbb{K}$ includes the presence of production excesses as in $\widetilde{\mathbb{K}}$.

Following the previous remarks, we can replace the production costs and the storage costs as

$$
f_{i}^{k}(x)=\widetilde{f}_{i}^{k}(x, \varepsilon), \quad g_{i}^{k}(x)=\widetilde{g}_{i}^{k}(x, \varepsilon), \quad \forall i=1, \ldots, m, \forall k=1, \ldots, m .
$$


Similarly, we can express the profit of firm $P_{I}$ as

$$
v_{i}(x)=\widetilde{v}_{i}(x, \varepsilon)=\sum_{k=1}^{l}\left[\sum_{j=1}^{n} d_{j}^{k}(x) x_{i j}^{k}-f_{i}^{k}(x)-g_{i}^{k}(x)-\sum_{j=1}^{n} c_{i j}^{k}(x) x_{i j}^{k}\right],
$$

The noncooperative peculiarity enters in our model since firm tries to maximize its own profit function considering the optimal distribution pattern of the others. Our aim is to determine the "best" commodity distribution for all the actors in the model, i.e. a nonnegative tensor commodity distribution $x$ for which the $m$ firms and the $n$ demand markets will be in a suitable state of equilibrium. We state below a definition of equilibrium which fits the model and generalizes the Cournot-Nash equilibrium principle.

Definition 4.1. A feasible tensor distribution $x^{*} \in \mathbb{K}$ is an oligopolistic market equilibrium in presence of production excesses if and only if, for each $i=1, \ldots, m$, it results

$$
v_{i}\left(x^{*}\right) \geq v_{i}\left(x_{i}, x_{-i}^{*}\right),
$$

where $x_{-i}^{*}=\left(x_{1}^{*}, \ldots, x_{i-1}^{*}, x_{i+1}^{*}, \ldots, x_{m}^{*}\right)$ and $x_{i}$ is a slice of dimension $n l$.

We indicate with

$$
\nabla_{D^{v}}=\left(\frac{\partial v_{i}}{\partial x_{i j}^{k}}\right)_{i j k}, \quad i=1, \ldots, m, j=1, \ldots, n, k=1, \ldots, l,
$$

the tensor of partial derivative of $v_{i}$ with respect to the tensor variable $x_{i j}^{k}, i=1, \ldots, m, j=$ $1, \ldots, n, k=1, \ldots, l$.

We need the following assumptions to derive an equivalent formulation of Definition 4.1 with a suitable tensor variational inequality. In the sequel, let us assume the following hypotheses:

(i) $v_{i}(x)$ is continuously differentiable with respect to $x$, for each $i=1, \ldots, m$;

(ii) $v_{i}(x)$ is pseudoconcave with respect to the variables $x \in \mathscr{T}$, namely, the following condition holds (see [1])

$$
\begin{aligned}
& \left\langle\frac{\partial v_{i}}{\partial x_{i}}\left(x_{1}, \ldots, x_{i}, \ldots, x_{m}\right), x_{i}-y_{i}\right\rangle \geq 0 \\
& \quad \Rightarrow v_{i}\left(x_{1}, \ldots, x_{i}, \ldots, x_{m}\right) \geq v_{i}\left(x_{1}, \ldots, y_{i}, \ldots, x_{m}\right) .
\end{aligned}
$$

Under above assumptions on $v_{i}$, we establish the following equilibrium formulation.

Theorem 4.1. Let us suppose that assumptions (i) and (ii) are satisfied. Then, $x^{*} \in \mathbb{K}$ is an oligopolistic market equilibrium in presence of production excesses according to Definition 4.1 if and only if it satisfies the following tensor variational inequality

$$
\begin{aligned}
& \left\langle-\nabla_{D} v\left(x^{*}\right), x-x^{*}\right\rangle \\
& =-\sum_{i=1}^{m} \sum_{j=1}^{n} \sum_{k=1}^{l} \frac{\partial v_{i}\left(x^{*}\right)}{\partial x_{i j}^{k}}\left(x_{i j}^{k}-\left(x_{i j}^{k}\right)^{*}\right) \geq 0, \quad \forall x \in \mathbb{K} .
\end{aligned}
$$

Proof. First, let us prove that if $x^{*}$ satisfies (4.6), then it is a general oligopolistic market equilibrium point. By contradiction, let us suppose that there exists $i^{*}$ such that

$$
v_{i^{*}}\left(x^{*}\right)<v_{i^{*}}\left(x_{i^{*}}, x_{-i^{*}}^{*}\right) .
$$




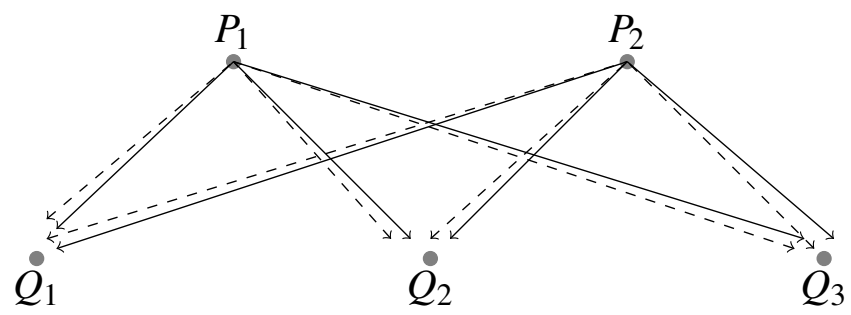

FIGURE 2. Network structure of the oligopoly

Since $v(x)$ is a pseudoconcave function, it follows that

$$
\left\langle-\nabla_{D} v\left(x^{*}\right), x-x^{*}\right\rangle<0 .
$$

The opposite implication follows straightforwardly.

By using the theoretical results proved in the previous sections for tensor variational inequalities and taking into account that the feasible set $\mathbb{K}$ is nonempty convex compact, we can now derive a theorem of existence and uniqueness for the general oligopolistic market equilibrium distribution.

Theorem 4.2. Suppose that assumptions (i) and (ii) are satisfied. Moreover, if $-\nabla_{D} v$ is strictly monotone, then the general oligopolistic market equilibrium distribution is unique.

\section{THE NUMERICAL EXAMPLE}

Let us describe here a numerical example of the general oligopolistic market equilibrium problem in presence of production excesses in which two firms $P_{1}$ and $P_{2}$ compete with three markets $Q_{1}, Q_{2}$ and $Q_{3}$. Every firm $P_{i}, i=1,2$, produces two different kinds of commodities. Figure 2 shows the network structure: dashed and continuous lines represent the two kinds of commodities. Let $x_{i j}^{k}$ be the $k$-th commodity shipment from $P_{i}$ to $Q_{j},(i=1,2, j=1, \ldots, 3, k=$ $1,2)$ and assume that the constraint $0 \leq x_{i j}^{k} \leq 100$ holds, for every $i=1,2, j=1, \ldots, 3, k=1,2$.

Let $p$ be the matrix of the commodity production:

$$
p=\left(\begin{array}{ll}
1 & 2 \\
2 & 1
\end{array}\right)
$$

As a consequence, the feasible set is

$$
\begin{aligned}
\mathbb{K}= & \left\{x \in \mathscr{T}\left(\mathbb{R}_{+}\right):\right. \\
& 0 \leq x_{i j}^{k} \leq 100, \quad \forall i=1,2, \forall j=1,2,3, \forall k=1,2 \\
& \left.\sum_{j=1}^{3} x_{i j}^{k} \leq p_{i}, \quad \forall i=1,2, \forall k=1,2\right\} .
\end{aligned}
$$

Let us consider the profit functions $v_{i}$ defined by

$$
\begin{aligned}
v_{1}= & -4\left(x_{11}^{1}\right)^{2}-4\left(x_{12}^{1}\right)^{2}-6\left(x_{13}^{1}\right)^{2}-6\left(x_{11}^{2}\right)^{2}-5\left(x_{21}^{1}\right)^{2}-2\left(x_{22}^{1}\right)^{2} \\
& -4 x_{11}^{1} x_{12}^{1}-6 x_{13}^{1} x_{11}^{2}-2 x_{21}^{1} x_{22}^{1}+3 x_{11}^{1}+4 x_{12}^{1}+x_{13}^{1}+x_{11}^{2}+2 x_{21}^{1}+2 x_{22}^{1},
\end{aligned}
$$


102

$$
\begin{aligned}
v_{2}= & -10\left(x_{22}^{2}\right)^{2}-4\left(x_{23}^{2}\right)^{2}-4\left(x_{13}^{2}\right)^{2}-5\left(x_{12}^{2}\right)^{2}-2\left(x_{23}^{1}\right)^{2}-2\left(x_{21}^{2}\right)^{2} \\
& -2 x_{11}^{1} x_{23}^{2}-2 x_{13}^{2} x_{12}^{2}-2 x_{23}^{1} x_{21}^{2}+x_{22}^{2}+2 x_{23}^{2}+10 x_{13}^{2}+3 x_{12}^{2}+3 x_{23}^{1}+2 x_{21}^{2} .
\end{aligned}
$$

Then, the components of $\nabla_{D} v$ different from zero are given by

$$
\begin{array}{ll}
\frac{\partial v_{1}}{x_{11}^{1}}=-8 x_{11}^{1}-4 x_{12}^{1}+3, & \frac{\partial v_{2}}{x_{23}^{1}}=-4 x_{23}^{1}-2 x_{21}^{2}+3 \\
\frac{\partial v_{1}}{x_{12}^{1}}=-4 x_{11}^{1}-8 x_{12}^{1}+4, & \frac{\partial v_{2}}{x_{21}^{2}}=-2 x_{23}^{1}-4 x_{21}^{2}+2 \\
\frac{\partial v_{1}}{x_{13}^{1}}=-12 x_{13}^{1}-6 x_{11}^{2}+1, & \frac{\partial v_{2}}{x_{22}^{2}}=-20 x_{22}^{2}-2 x_{12}^{1}+1, \\
\frac{\partial v_{1}}{x_{11}^{2}}=-6 x_{13}^{1}-12 x_{11}^{2}+1, & \frac{\partial v_{2}}{x_{23}^{2}}=-8 x_{23}^{2}-2 x_{11}^{1}+2, \\
\frac{\partial v_{1}}{x_{21}^{1}}=-10 x_{21}^{1}-2 x_{22}^{1}+2, & \frac{\partial v_{2}}{x_{13}^{2}}=-8 x_{13}^{2}-2 x_{12}^{2}+10 \\
\frac{\partial v_{1}}{x_{22}^{1}}=-2 x_{21}^{1}-4 x_{22}^{1}+2, & \frac{\partial v_{2}}{x_{12}^{2}}=-2 x_{13}^{2}-10 x_{12}^{1}+3 .
\end{array}
$$

By Theorem 4.1, the general oligopolistic market equation distribution is a solution to

$$
\begin{aligned}
& \left\langle-\nabla_{D} v\left(x^{*}\right), x-x^{*}\right\rangle \\
& \quad=-\sum_{i=1}^{2} \sum_{j=1}^{3} \sum_{k=1}^{2} \frac{\partial v_{i}\left(x^{*}\right)}{\partial x_{i j}^{k}}\left(x_{i j}^{k}-\left(x_{i j}^{k}\right)^{*}\right) \geq 0, \quad \forall x \in \mathbb{K} .
\end{aligned}
$$

In order to compute the solution to (5.2), we make use of the direct method proposed in [16]. More precisely, we consider the following system:

$$
\left\{\begin{array}{l}
8 x_{11}^{1}+4 x_{12}^{1}-3=0 \\
12 x_{13}^{1}+6 x_{11}^{2}-1=0 \\
10 x_{21}^{1}+2 x_{22}^{1}-2=0 \\
4 x_{23}^{1}+2 x_{21}^{2}-3=0 \\
20 x_{22}^{2}+2 x_{12}^{1}-1=0 \\
8 x_{13}^{2}+2 x_{12}^{2}-10=0 \\
4 x_{11}^{1}+8 x_{12}^{1}-4=0 \\
6 x_{13}^{1}+12 x_{11}^{2}-1=0 \\
2 x_{21}^{1}+4 x_{22}^{1}-2=0 \\
2 x_{23}^{1}+4 x_{21}^{2}-2=0 \\
8 x_{23}^{2}+2 x_{11}^{1}-2=0 \\
2 x_{13}^{2}+10 x_{12}^{1}-3=0
\end{array}\right.
$$


and we compute the solution $x^{*}$ :

$$
\begin{aligned}
& \left(x_{11}^{1}\right)^{*}=1 / 6, \\
& \left(x_{12}^{1}\right)^{*}=5 / 12, \\
& \left(x_{13}^{1}\right)^{*}=1 / 18, \\
& \left(x_{11}^{2}\right)^{*}=1 / 18, \\
& \left(x_{21}^{1}\right)^{*}=1 / 9, \\
& \left(x_{22}^{1}\right)^{*}=4 / 9, \\
& \left(x_{23}^{1}\right)^{*}=2 / 3, \\
& \left(x_{21}^{2}\right)^{*}=1 / 6, \\
& \left(x_{22}^{2}\right)^{*}=1 / 120, \\
& \left(x_{23}^{2}\right)^{*}=5 / 24, \\
& \left(x_{13}^{2}\right)^{*}=47 / 38, \\
& \left(x_{12}^{2}\right)^{*}=1 / 19 .
\end{aligned}
$$

Observing that $x^{*}$ verifies the feasible conditions, we can deduct that it is the general oligopolistic market equilibrium distribution. Finally, we obtain the production excesses:

$$
\varepsilon=\left(\begin{array}{cc}
11 / 72 & 112 / 171 \\
7 / 9 & 37 / 60
\end{array}\right)
$$

\section{Conclusions}

In the paper, we obtained some results on tensor variational inequalities and presented some projection algorithms to solve such inequalities. More precisely, we proved some properties and a fixed point theorem for solutions to such inequalities. We introduced also some numerical methods and established that the sequences determined by such methods convergence to solutions. In the second part of the paper, we considered a general oligopolistic market equilibrium model in which each firm produces several commodities and some production excesses occur. The equilibrium distribution is equivalent to the solution to a suitable tensor variational inequality. Thanks to the variational formulation of the model, we applied the theoretical results obtained in the first part. Finally a numerical example is provided.

\section{Acknowledgments}

The first and second authors were partially supported by PRIN 2017 Nonlinear Differential Problems via Variational, Topological and Set-valued Methods (Grant 2017AYM8XW) and by INdAM GNAMPA Project 2019 Tecniche variazionali in problemi di ottimizzazione. The third author was partially supported by Gruppo Nazionale per il Calcolo Scientifico - Istituto Nazionale di Alta Matematica (GNCS-INdAM).

\section{REFERENCES}

[1] C. Baiocchi, A. Capelo, Variational and Quasivariational Inequalities, Applicationsto Free Boundary Problems, John Wiley Sons, New York, 1984. 
[2] A. Barbagallo, S. Guarino Lo Bianco, Variational inequalities on a class of a structured tensors, J. Nonlinear Convex Anal. 19 (2018), 711-729.

[3] A. Barbagallo, S. Guarino Lo Bianco, On ill-posedness and stability of tensor variational inequalities: application to an economic equilibrium, J. Glob. Optim. (2019), https://doi.org/10.1007/s10898-019-00788-9.

[4] D.P. Bertsekas, J.N. Tsitsiklis, Parallel and Distribuited Computation, Numerical Method, Prentice-Hall, London 1989.

[5] M. Che, L. Qi, Y. Wei, Positive definite tensors to nonlinear complementarity problems, J. Optim. Theory and Appl. 168 (2016), 475-487.

[6] A. Cournot, Researches into the mathematical principles of the theory of wealth, MacMillan, London, 1897.

[7] S. Dafermos, A. Nagurney, Oligopolistic and competitive behavior of spatially separated markets, Regional Science and Urban Economics 17 (1987), 245-254.

[8] S.V. Denisov, V.V. Semenov, L.M. Chabak, Convergence of the modified extragradient method for variational inequalities with non-Lipschitz iperators, Cybern. Syst. Anal. 51 (2015), 757-765.

[9] Q.L. Dong, Y.Y. Lu, J. Yang, The extragradient algorithm with inertial effects for solving the variational inequality, Optimization 65 (2016), 2217-2226.

[10] F. Facchinei, J.S. Pang, Finite-Dimensional Variational Inequalities and Complementarity Problems, Volume I and Volume II, Springer-Verlag, New York, 2003.

[11] D. Gabay, H. Moulin, On the Uniqueness and Stability of Nash Equilibria in Noncooperative Games, In: A. Bensousson et al., (eds.) Applied Stochastic Control in Econometrics and Management Science, pp.271-293, North Holland, Amsterdam, 1980.

[12] S. Karamardian, Non-linear complementary problem with applications, Part 1, J. Optim. Theory Appl. 4 (1969), 87-98.

[13] S. Karamardian, Non-linear complementary problem with applications, Part 2, J. Optim. Theory Appl. 4 (1969), 167-135.

[14] E.N. Khobotov, Modification of the extra-gradient method for solving variational inequalities certain optimization problems, USSR Comput. Math. Math. Phys. 27 (1987), 1462-1473.

[15] P. Marcotte, Application of Khobotov's algorithm to variational inequalities and network equilibrium problems, Inform. Syst. Oper. Res. 29 (1991), 258-270.

[16] A. Maugeri, Convex programming, variational inequalities and applications to the traffic equilibrium problem, Appl. Math. Optim. 16 (1987), 169-185.

[17] Y.V. Malitsky, Projected reflected gradient method for variational inequalities, SIAM J. Optim. 25 (2015), $502-520$.

[18] M.A. Noor, Some developments in general variational inequalities, Appl. Math. Comput. 152 (2004), 199277.

[19] J.F. Nash, Equilibrium points in $n$-person games, Proc. Natl. Acad. Sci. USA 36 (1950), 48-49.

[20] J.F. Nash, Non-cooperative games, Ann. Math. 54 (1951), 286-295.

[21] X. Qin, J.C. Yao, A viscosity iterative method for a split feasibility problem, J. Nonlinear Convex Anal. 20 (2019), 1497-1506.

[22] J.B. Rosen, Existence and uniqueness of equilibrium points for concave $n$-person games, Econometrica 33 (1965), 520-533.

[23] Y. Song, L. Qi, Properties of some classes of structured tensors, J. Optim. Theory and Appl. 165 (2015), 854-873.

[24] M. Sibony, Méthodes itératives pour les équations et inéquations aux dérivées partielles non linéaires de type monotone, Calcolo 7 (1970), 65-183.

[25] P. Tseng, On linear convergence of iterative methods for the variational inequality problem, J. Comput. Appl. Math. 60 (1995), 237-252.

[26] M. Tian, M. Tong, A self-adaptive Armijo-like step size method for solving monotone variational inequality problems in Hilbert spaces, J. Nonlinear Funct. Anal. 2019 (2019), 1-15.

[27] Y. Wang, Z. Huang, L. Qi, Introduction to tensor variational inequalities, arXiv:1701.07677.

[28] Q. Yang, On variable-step relaxed projection algorithm for variational inequalities, J. Math. Anal. Appl. 302 (2005), 166-179. 
[29] E. Zeidler, Nonlinear Functional Analysis and Its Applications III: Variational Methods and Optimization, Springer-Verlag, New York, 1985. 